\title{
Ruptured Abdominal Aortic Aneurysm in a Patient with Congenital Fused Pelvic Kidney: A Case Report of Emergency Endovascular Treatment
}

\author{
Claudio Bianchini Massoni, MD ${ }^{1}$ Matteo Azzarone, MD ${ }^{1}$ Danilo Barbarisi, MD ${ }^{1}$ Paolo Perini, MD ${ }^{1}$ \\ Antonio Freyrie, $\mathrm{MD}^{1}$ \\ 1 Vascular Surgery - Department of Surgical Sciences, \\ Azienda Ospedaliero-Universitaria di Parma, Parma, Italy \\ Address for correspondence Claudio Bianchini Massoni, MD, Vascular \\ Surgery - Department of Surgical Sciences, Institution: Azienda \\ Ospedaliero-Universitaria di Parma, Via Gramsci 14 - 43126 Parma \\ AORTA 2018;6:21-27. \\ (PR), Italy (e-mail: claudiobianchinim@gmail.com).
}
Abstract
Keywords
- fused pelvic kidney
- abdominal aortic aneurysm
- aortic rupture
- endovascular procedures

\begin{abstract}
A 90-year-old male developed acute onset of abdominal and lumbar pain due to the rupture of an $11-\mathrm{cm}$ abdominal aortic aneurysm. A congenital fused pelvic kidney perfused by three renal arteries arising from iliac axes was detected. In an emergent setting, an aorto-uni-iliac endograft was deployed through right femoral surgical access with occlusion of the upper right renal artery. An occluder device was placed in the common iliac artery above the renal artery through left femoral access. A femorofemoral crossover bypass completed the procedure. The patient developed acute renal failure, with no dialysis necessity. One-month computed tomography angiography showed procedure success.
\end{abstract}

\section{Introduction}

Congenital renal abnormalities occur in 3.3 to $11 \%$ of the population $^{1}$ and include alterations in the number, shape, and/or site of the organ. ${ }^{2}$ In patients with abdominal aortic aneurysm (AAA), the incidence of congenital renal variants is not well known. In a case series over a 20 -year period, AAAs with congenital kidney anomalies were seen in 25 patients. $^{3}$

In this population, the number and site of origin of renal arteries are extremely variable and, for this reason, the treatment of AAA is more complex. Assessment of renal arteries (dimension, stenosis, and thrombus) and renal function (creatinine, serum urea level, and glomerular filtration rate) is mandatory for optimal planning and to obtain technical and clinical success after any type of procedure (surgical, endovascular, and hybrid).

Management of ruptured AAA is challenging and perioperative mortality after emergency open or endovascular treatment reaches $53 \%{ }^{4}$

The aim of this article is to report a case of emergency endovascular treatment of a ruptured AAA with congenital fused pelvic kidney (CFPK).

\section{Case Presentation}

A 90-year-old male presented to the emergency department complaining of severe abdominal and back pain. The symptoms began 3 hours earlier. The patient was severely obese and under chronic pharmacological therapy for arterial hypertension and coronary artery disease (previous coronary artery bypass graft). The abdominal computed tomography angiogram (CTA) showed an 11-cm AAA with a retroperitoneal hematoma and a posterior wall rupture (-Fig. 1). The aortic rupture was partially contained by the vertebral bodies and retroperitoneum. A unique lobulated renal mass was detected in pelvic region (CFPK or pancake kidney ${ }^{2}$ ) (-Fig. 2). Three renal arteries were detected arising from both iliac axes: one ( $8 \mathrm{~mm}$ in diameter) from the proximal portion of the right common iliac artery, the second ( $9 \mathrm{~mm}$ in diameter) from the right hypogastric artery, and the third ( $4 \mathrm{~mm}$ in diameter) from the distal segment of the left common iliac artery (-Fig. 3). The proximal neck (between the superior mesenteric artery and the AAA) was $20 \mathrm{~mm}$ in length and 23 to

Copyright (c) 2018 by Thieme Medical Publishers, Inc., 333 Seventh Avenue, New York, NY 10001, USA. Tel: +1(212) 584-4662.
License terms

(ब)(1) 10.1055/s-0038-1636992. ISSN 2325-4637. 


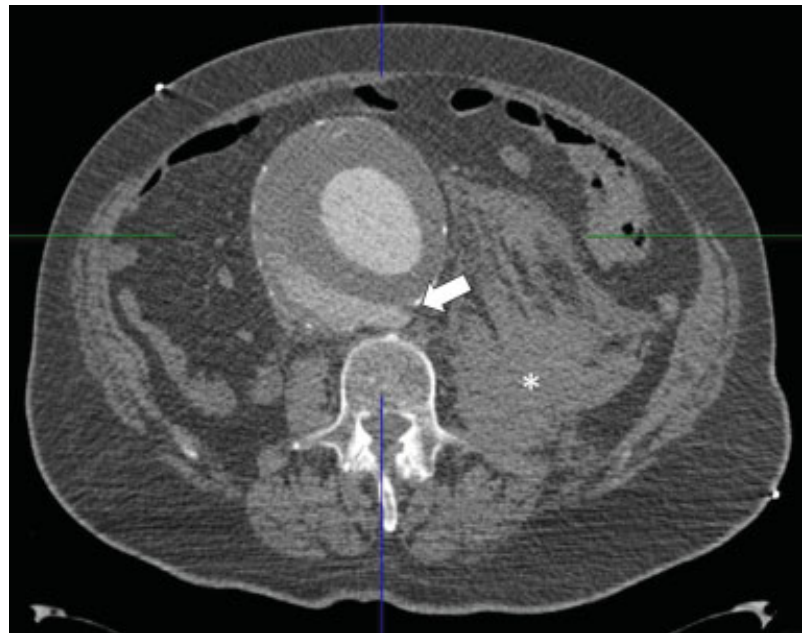

Fig. 1 Computed tomography angiogram showing a voluminous abdominal aortic aneurysm (11.5 cm in diameter) with rupture of posterior aortic wall (white arrow) and intra-abdominal hematoma (white asterisk) on the left retroperitoneal region.

$24 \mathrm{~mm}$ in diameter with no intraluminal thrombus or wall calcification. The patient was conscious and hemodynamically stable (systolic blood pressure: $90-100 \mathrm{~mm} \mathrm{Hg}$ ). Considering the presence of a favorable proximal aortic neck, the partial hemodynamic stability, the age, and the obesity, an endovascular treatment was preferred. Only aorto-uni-iliac endografts were available for emergency procedures.
Preoperative planning was performed using 3mensio Vascular (Pie Medical Imaging) during patient transport to the operating theater and anesthetic induction.

Under general anesthesia, inguinal longitudinal cut-down was performed bilaterally and the common femoral arteries were isolated. An Endurant aorto-uni-iliac endograft (Medtronic Endovascular) was introduced through the right access. The C-arm was rotated at $50^{\circ}$-left anterior oblique and the preoperative angiogram was performed. The endograft was deployed below the origin of superior mesenteric artery, using the inframesenteric nondilated aorta as proximal sealing zone ( $\sim 20 \mathrm{~mm}$ in length). Two Endurant endograft extensions (Medtronic) were necessary to obtain sufficient sealing at the right common iliac artery with the occlusion of the upper renal artery. Through the left access, a Talent occluder was deployed in the proximal left common iliac artery, above the origin of left renal artery. The completion angiogram showed patency of the endografts and no endoleak from left iliac axis or renal or lumbar arteries (-Fig. 4). The procedure was completed with an extra-anatomical femorofemoral suprapubic bypass graft ( $8 \mathrm{~mm}$-Silver Dacron). Operative procedure time was 220 minutes and $120 \mathrm{cc}$ of contrast agent was injected (iomeprol, Iomeron 300; Bracco).

Ventilator support was removed after 24 hours and the abdominal and lumbar pain disappeared. Acute renal failure developed ( $\mathbf{F i g} . \mathbf{5}^{5}$ ) but no dialysis was necessary. The patient returned to the ward on the 9th postoperative day and started mobilization. The postoperative duplex ultrasound showed endograft patency with no endoleak. Renal
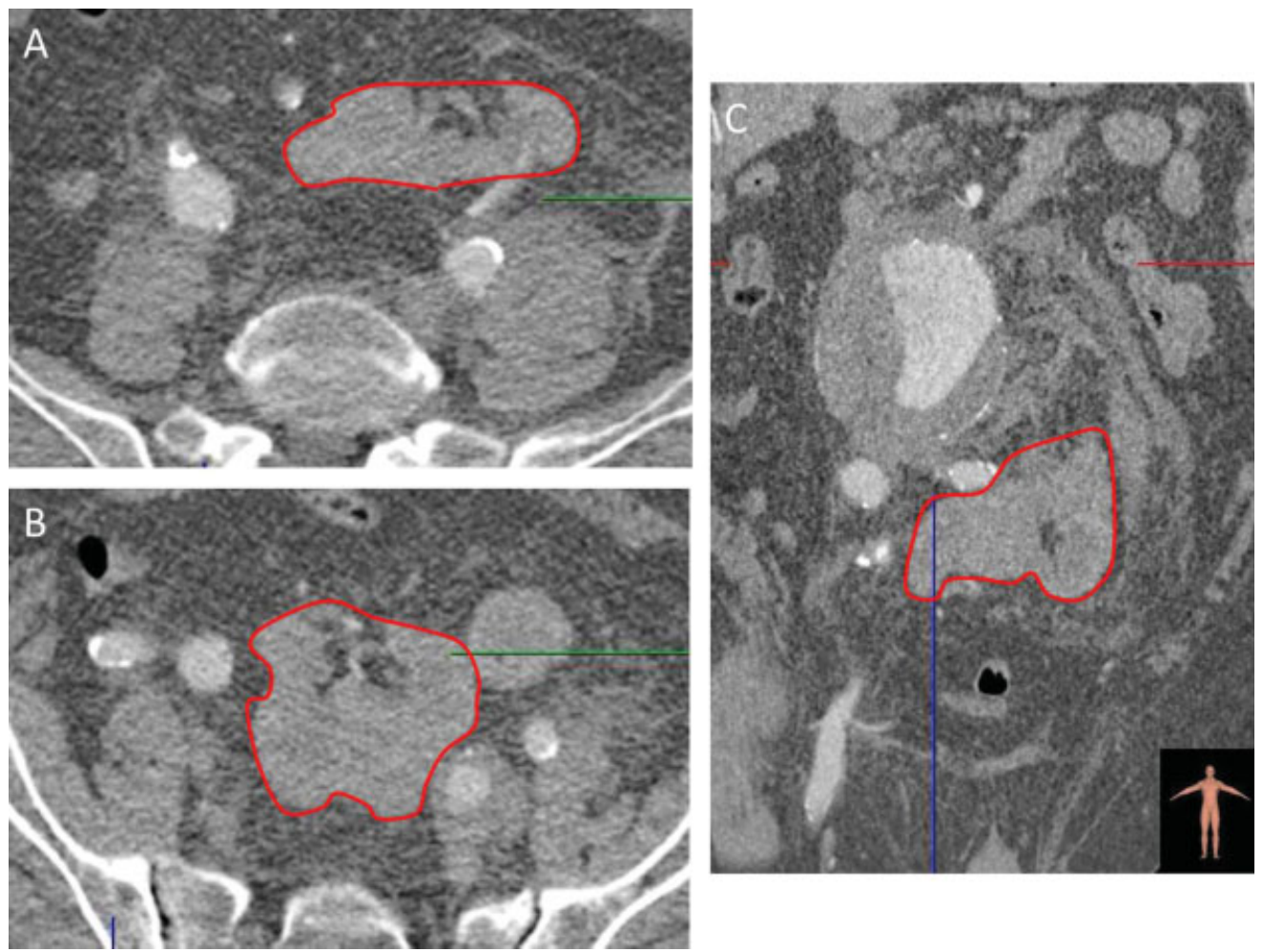

Fig. 2 Computed tomography angiogram imaging of congenital fused pelvic kidney or pancake kidney. (A and B) axial images; red line: renal parenchyma. (C) Multiplanar reconstruction; red line: renal parenchyma. 

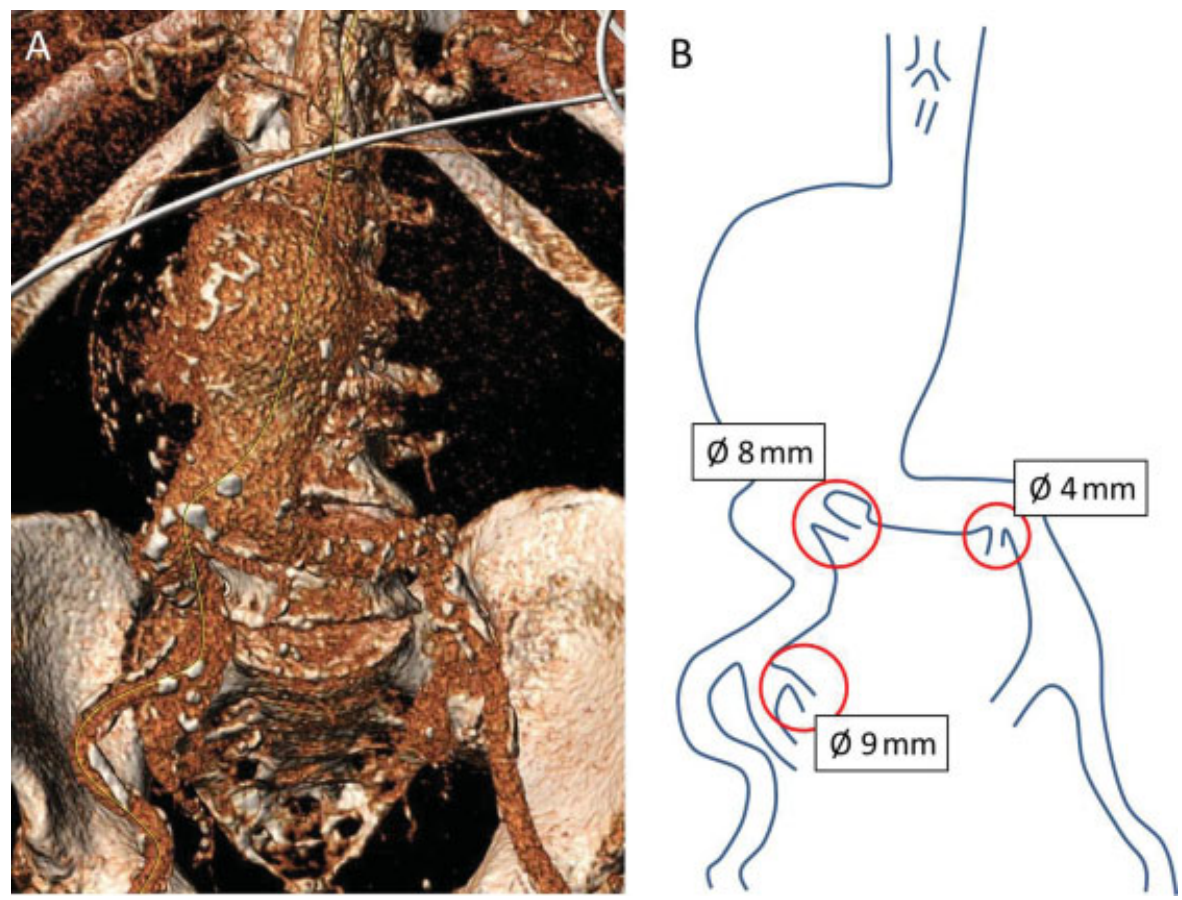

Fig. 3 (A) Volume rendering reconstruction of preoperative computed tomography angiogram (anteroposterior view). (B) Aortic outline and origin of renal arteries.

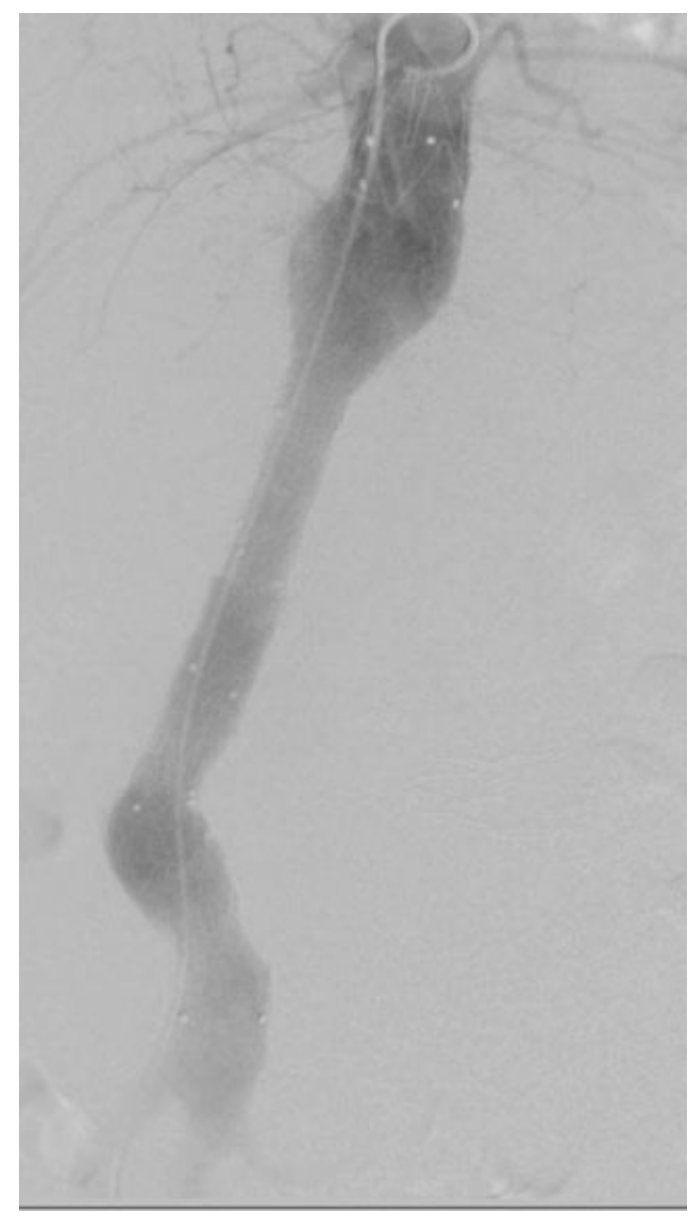

Fig. 4 Anteroposterior digital subtraction angiogram after deployment of aortouniiliac endograft, two iliac extensions (through right femoral access) and occluder (through left femoral access); no endoleaks are detected. function gradually improved (-Fig. 5) and the patient was discharged on the 12 th postoperative day.

At 1 month, the renal function remained mildly altered (creatinine serum level: $1.9 \mathrm{mg} / \mathrm{dL}$ ). After intravenous hydration, the abdominal CTA showed patency of the aorto-uniiliac endograft and femorofemoral bypass graft, sac shrinkage, and no evidence of endoleaks. The pelvic kidney presented no ischemic lesions and was perfused by two renal arteries (lower right and left) (-Fig. 6).

\section{Discussion}

Congenital pelvic kidney (CPK) is defined as the presence of one ectopic kidney low lying in the abdomen. ${ }^{2}$ This variant has been reported to be present in 1 to 2,200 to 1 in 3,000 individuals. $^{6}$

Congenital fused pelvic kidney, also called "pancake kidney," is a rare congenital anatomic variant consisting of unique lobulated pelvic renal mass of dual parenchymatous systems. ${ }^{2}$ During autopsies, this variant is observed in 1 out 22,000 cases. $^{7}$

Considering patients who underwent major aortic surgery, $\sim 0.18 \%$ presented a CPK. ${ }^{8}$ Thus, the association between AAA and CFPK is extremely rare.

These two anatomical variants are often associated with multiple aberrant renal arteries arising from the aortic bifurcation and iliac arteries. AAA treatment is technically challenging, and has a higher risk of kidney failure.

In the current case, endovascular treatment was preferred because of the advanced age and the severe obesity of the patient.

No bifurcated endografts were available in our center for procedures in an urgent/emergent setting, so aorto-uni-iliac 

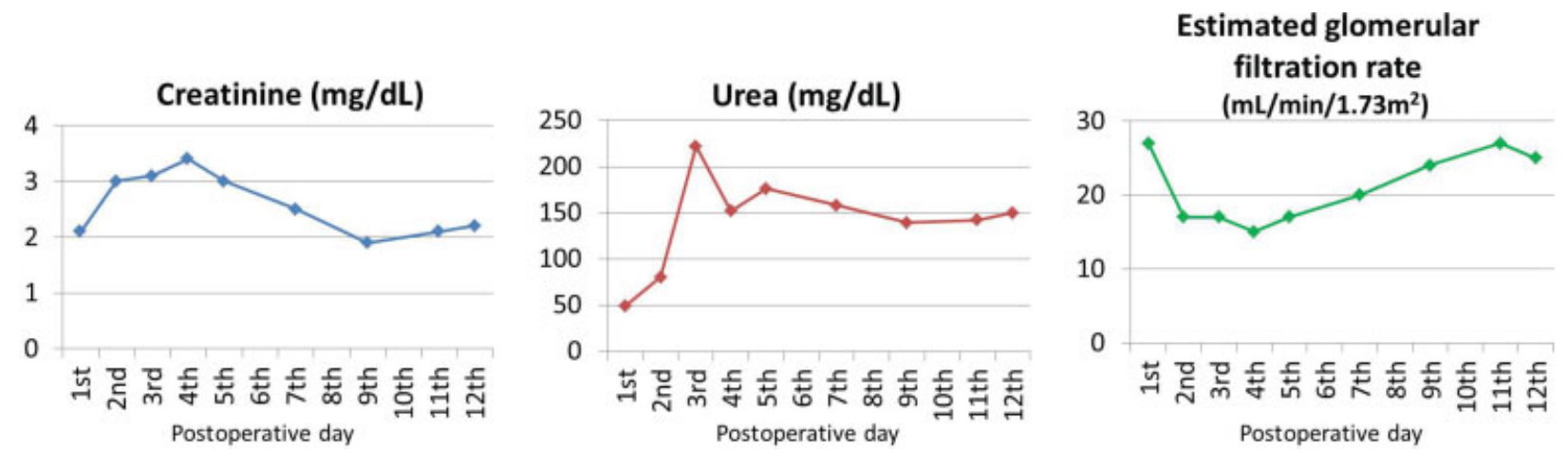

Fig. 5 Serum levels of creatinine, urea, and estimated glomerular filtration rate (according to the Chronic Kidney Disease Epidemiology Collaboration Eq. ${ }^{5}$ ) during hospitalization.
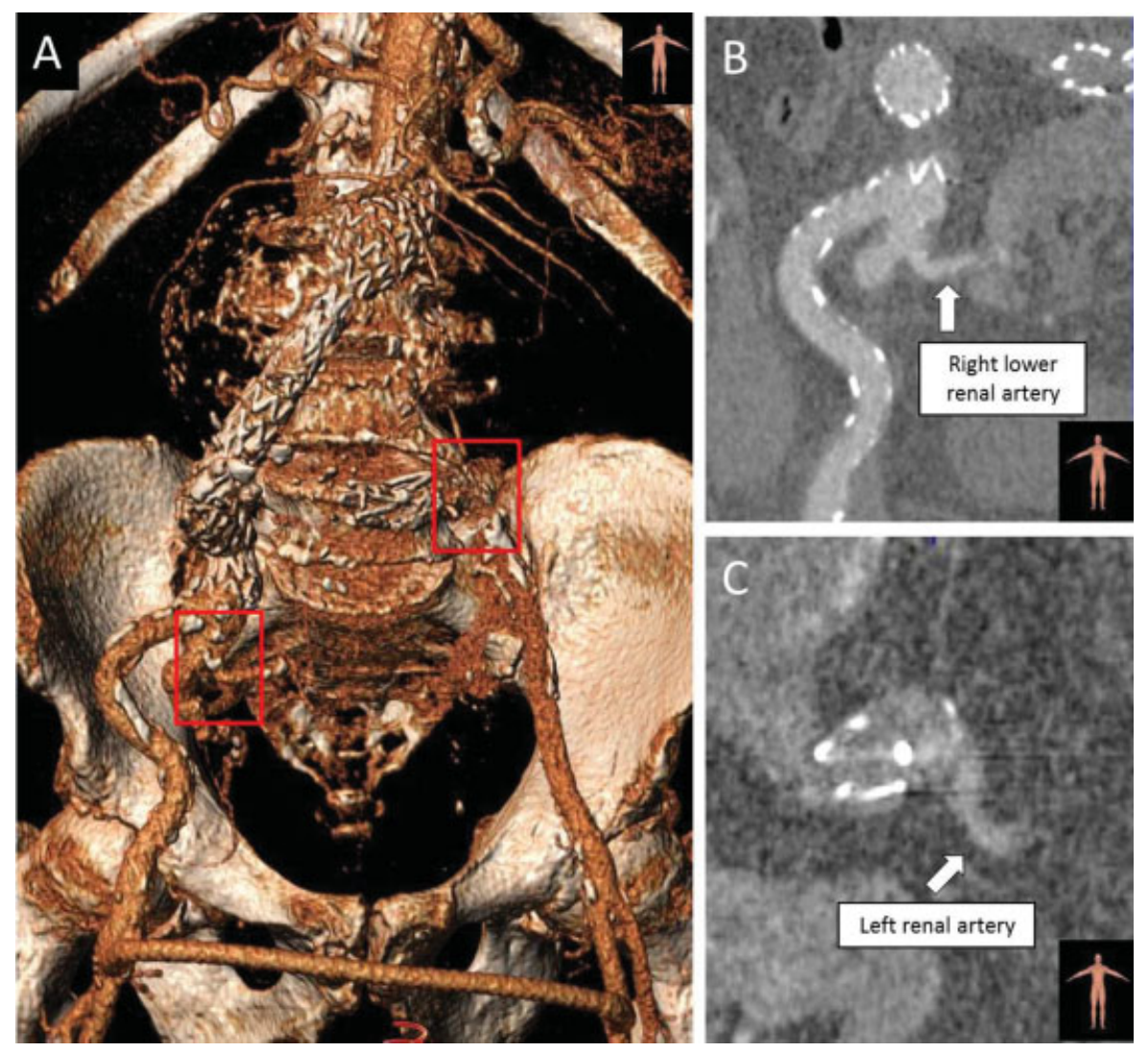

Fig. 6 One-month computed tomography angiogram. (A) Volume rendering shows patency of celiac trunk, superior mesenteric artery, endograft, and femorofemoral crossover bypass. (B) Patency of lower right renal artery (arising from right hypogastric artery) (white arrow). (C) Patency of renal artery arising from left common iliac artery, below occluder device (white arrow).

endografting and femorofemoral bypass were obligatory choices. Considering the sites of origin of the three renal arteries, the coverage of upper right renal artery was inevitable to obtain an adequate distal sealing zone. Also in case of left-side deployment of the aorto-uni-iliac endograft, the upper right renal artery would have been excluded by the occluder device deployment in the right common iliac artery.

To reduce the risk of persistent type 2 endoleak, a sac embolization may be intraoperatively performed. This adjunctive procedure was not executed to reduce time of intervention and was not considered strictly necessary.
Indeed, no patent lumbar arteries had been detected at preoperatory CTA, and the upper right renal artery arises from a nondilated segment making unlikely a back flow into the aneurysmal sac.

In case of renal anomalies, assessment of preoperative CTA is essential to define their number, origin, and size. In the literature, sacrifice of accessory renal arteries with diameter $<3 \mathrm{~mm}$ is considered noninfluential on renal function. ${ }^{9}$ In the current case, we excluded an $8-\mathrm{mm}$ renal artery, sparing the other two renal arteries $(9 \mathrm{~mm}$ and $4 \mathrm{~mm}$ in diameter). Transient renal function worsening was an expected event. 


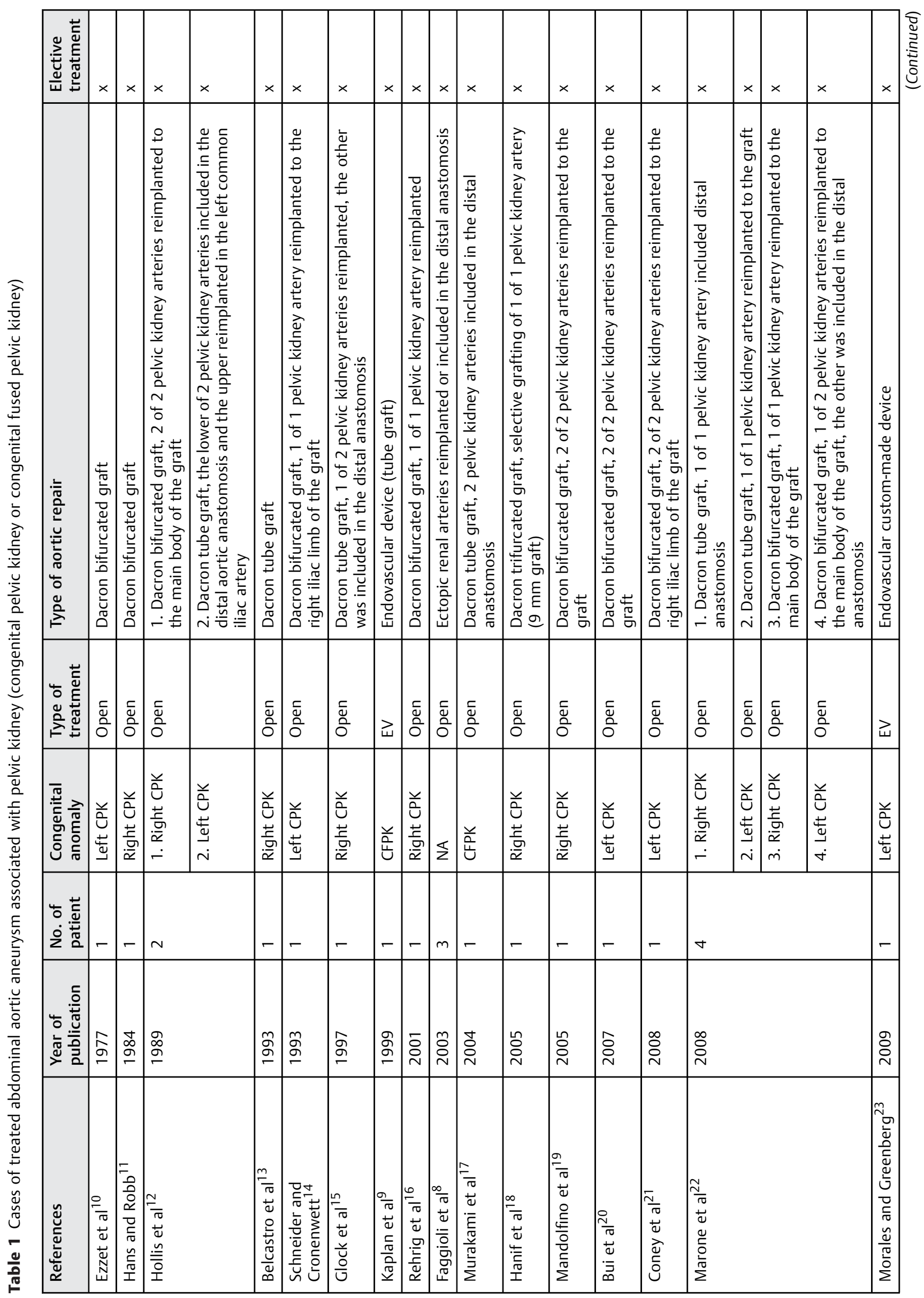




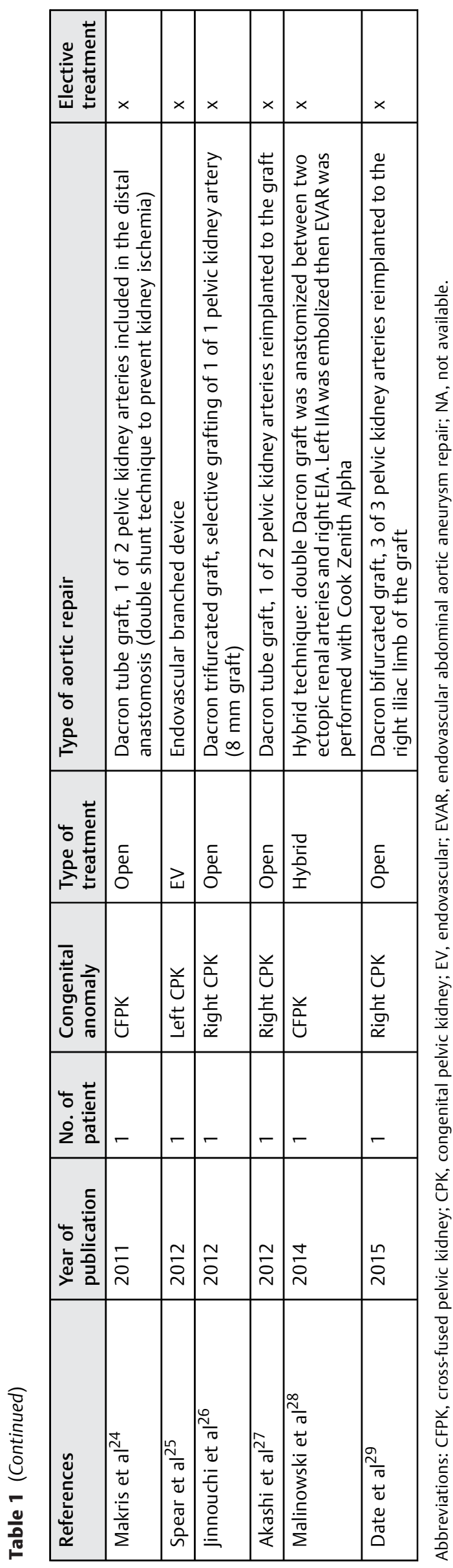

Ultimately, no dialytic treatment was required. After 1 month, mild renal insufficiency persisted.

Several cases of treatment of AAA associated with CPK or CFPK have been reported in literature ( - Table 1$) \cdot{ }^{8-29}$ To our knowledge, the current case is the first reporting of the treatment of a ruptured AAA associated with the presence of CFPK.

Surgical treatment of AAA in patients with pelvic kidney (CPK or CFPK) is more complex than in the general AAA population. Perfusion of the kidney must be maintained during aortic clamping and graft sewing. Renal arteries with diameter $>3 \mathrm{~mm}$ should be reimplanted ${ }^{9}$ and perfusion with cold Ringer solution is necessary in case of crossclamp time $>40$ minutes. $^{8}$

Total endovascular treatment has also been described. Morales and Greenberg ${ }^{23}$ treated a patient with a type IV thoracoabdominal aortic aneurysm associated with a left CPK, deploying a custom-made fenestrated endograft. A fenestration near the distal bifurcation of the endograft permitted the perfusion of the CPK through a covered bridging stent. Also, a parallel stent graft technique has been described to perfuse an ectopic renal artery originating from the AAA in a patient with a crossover ectopic right kidney fused with the left kidney. ${ }^{30}$ A Viabahn-Gore (W. L. Gore) endograft reinforced with balloon-expandable stents was deployed in a parallel fashion with an Endurant II-Medtronic endograft iliac limb.

Hybrid treatment has also been reported. In a case of CFPK with renal arteries arising from the AAA, a double iliac-renal bypass was primarily sutured and an aorto-bifurcated endograft was deployed to exclude the aneurysm. ${ }^{28}$

In conclusion, in case of CPK or CFPK, endovascular, open surgical, and hybrid treatments of AAA have been described in literature and are potentially feasible. The most suitable treatment must be chosen according to the patient's clinical condition and arterial anatomy. Our case demonstrates that accurate preoperative planning is vital to achieve technical success and to preserve renal function in this complex subset of patients.

\section{Conflict of Interest}

All authors certify that they have NO affiliations with or involvement in any organization or entity with any financial interest (such as honoraria; educational grants; participation in speakers' bureaus; membership, employment, consultancies, stock ownership, or other equity interest; and expert testimony or patent-licensing arrangements), or nonfinancial interest (such as personal or professional relationships, affiliations, knowledge, or beliefs) in the subject matter or materials discussed in this manuscript.

\section{Funding}

None.

\section{Acknowledgements}

None.

\section{References}

1 Muttarak M, Sriburi T. Congenital renal anomalies detected in adulthood. Biomed Imaging Interv J 2012;8(01):e7 
2 Srinivas MR, Adarsh KM, Jeeson R, Ashwini C, Nagaraj BR. Congenital anatomic variants of the kidney and ureter: a pictorial essay. Jpn J Radiol 2016;34(03):181-193

3 Giordanengo F, Vandone P, Pizzocari P, De Monti M, Mattioli A. [Abdominal aortic aneurysm and horseshoe kidney. Clinical cases] [in Italian]. Minerva Cardioangiol 1991;39(03):97-102

4 Peters AS, Hakimi M, Erhart P, et al. Current treatment strategies for ruptured abdominal aortic aneurysm. Langenbecks Arch Surg 2016;401(03):289-298

5 Levey AS, Stevens LA, Schmid CH, et al; CKD-EPI (Chronic Kidney Disease Epidemiology Collaboration). A new equation to estimate glomerular filtration rate. Ann Intern Med 2009;150(09): 604-612

6 Cinman NM, Okeke Z, Smith AD. Pelvic kidney: associated diseases and treatment. J Endourol 2007;21(08):836-842

7 Bauer S, Perlmutter A, Retik A. Anomalies of the upper urinary tract. In: Walsh PC, ed. Campbell's Urology. 6th ed. Philadelphia PA: WB Saunders; 1992:1357-1437

8 Faggioli G, Freyrie A, Pilato A, et al. Renal anomalies in aortic surgery: contemporary results. Surgery 2003;133(06):641-646

9 Kaplan DB, Kwon CC, Marin ML, Hollier LH. Endovascular repair of abdominal aortic aneurysms in patients with congenital renal vascular anomalies. J Vasc Surg 1999;30(03):407-415

10 Ezzet F, Dorazio R, Herzberg R. Horseshoe and pelvic kidneys associated with abdominal aortic aneurysms. Am J Surg 1977;134 (02):196-198

11 Hans SS, Robb HJ. Abdominal aortic aneurysm and pelvic kidney. J Cardiovasc Surg (Torino) 1984;25(02):176-178

12 Hollis HW Jr, Rutherford RB, Crawford GJ, Cleland BP, Marx WH, Clark JR. Abdominal aortic aneurysm repair in patients with pelvic kidney. Technical considerations and literature review. J Vasc Surg 1989;9(03):404-409

13 Belcastro S, Azzena GF, Gorini P, Fogli L, Pavanelli L. Abdominal aortic aneurysmectomy associated with a native iliac kidney. Case report. J Cardiovasc Surg (Torino) 1993;34(01):83-84

14 Schneider JR, Cronenwett JL. Temporary perfusion of a congenital pelvic kidney during abdominal aortic aneurysm repair. J Vasc Surg 1993;17(03):613-617

15 Glock Y, Blasevich R, Laghzaoui A, Roux D, Fournial G. Abdominal aortic aneurysm and congenital pelvic kidney. A rare association. Tex Heart Inst J 1997;24(02):131-133

16 Rehrig ST Jr, Goff JM Jr, Hadro NC, Gillespie DL, O'Donnell SD, Rich NM. Repair of bilateral iliac artery aneurysms associated with a congenital pelvic kidney. Vasc Surg 2001;35(06):463-467

17 Murakami T, Makino Y, Suto Y, Yasuda K. Abdominal aortic aneurysm repair in a patient with a congenital solitary pelvic kidney. A case report. J Cardiovasc Surg (Torino) 2004;45(05): 501-504

18 Hanif MA, Chandrasekar R, Blair SD. Pelvic kidney and aorto-iliac aneurysm-a rare association-case report and literature review. Eur J Vasc Endovasc Surg 2005;30(05):531-533

19 Mandolfino T, Canciglia A, D’Alfonso M, Mirenda F. [Aneurysm of the abdominal aorta associated with an ectopic pelvic kidney and abnormal vascularisation: a case report] [in Italian]. Chir Ital 2005;57(03):361-364

20 Bui TD, Wilson SE, Gordon IL, Fujitani RM, Carson J, Montgomery RS. Renal function after elective infrarenal aortic aneurysm repair in patients with pelvic kidneys. Ann Vasc Surg 2007;21(02):143-148

21 Coney PM, Shanmugan V, Raman S, Williams HD, Downing R. Chronic type B dissecting aortoiliac aneurysm repair complicated by congenital pelvic kidney. J Vasc Surg 2008;48(03):727-729

22 Marone EM, Tshomba Y, Brioschi C, Calliari FM, Chiesa R. Aortoiliac aneurysm associated with congenital pelvic kidney: a short series of successful open repairs under hypothermic selective renal perfusion. J Vasc Surg 2008;47(03):638-644

23 Morales JP, Greenberg RK. Customised stent graft for complex thoraco-abdominal aneurysm associated with congenital pelvic kidney. Eur J Vasc Endovasc Surg 2009;37(05):557-559

24 Makris SA, Kanellopoulos E, Chronopoulos A, Vrachliotis TG, Doundoulakis N. A double shunt technique for the prevention of ischaemia of a congenital, solitary, pelvic kidney during abdominal aortic aneurysm repair: a case report. J Med Case Reports 2011;5:92

25 Spear R, Maurel B, Sobocinski J, et al. Technical note and results in the management of anatomical variants of renal vascularisation during endovascular aneurysm repair. Eur J Vasc Endovasc Surg 2012;43(04):398-403

26 Jinnouchi K, Suenaga E, Fumoto H, Miho T. A case of abdominal aortic aneurysm with congenital pelvic kidney. Jpn J Vasc Surg 2012;21:829-832

27 Akashi H, Ishizaka T, Tanaka H, Masuda M, Matsumiya G. Abdominal aortic aneurysm with right ectopic kidney. Jpn J Cardiovasc Surg 2012;41:266-269

28 Malinowski MJ, Al-Nouri O, Hershberger R, Halandras PM, Aulivola $\mathrm{B}$, Cho JS. Abdominal aortic aneurysm associated with congenital solitary pelvic kidney treated with novel hybrid technique. Ann Vasc Surg 2014;28(06):1566.e7-1566.e10

29 Date K, Okada S, Ezure M, et al. Aortoiliac aneurysm with congenital right pelvic kidney. Heart Vessels 2015;30(03):420-425

30 Kfoury E, Almanfi A, Dougherty KG, Krajcer Z. Endovascular abdominal aortic aneurysm repair by means of the chimney technique in a patient with crossed fused renal ectopia. Tex Heart Inst J 2016;43(03):232-235 